\title{
Surface plasmon interferometer in silicon-on-insulator: novel concept for an integrated biosensor
}

\author{
Peter Debackere, Stijn Scheerlinck, Peter Bienstman, Roel Baets \\ Photonics Research Group, Dept. of Information Technology, Ghent University - IMEC \\ Sint-Pietersnieuwstraat 41, 9000 Gent, Belgium \\ Peter.Debackere@intec.UGent.be
}

\begin{abstract}
We propose a novel configuration for a highly integrated and highly sensitive optical biosensor. The basic element of the sensor is a surface plasmon interferometer consisting of a thin layer of gold embedded in a silicon membrane. We investigate the performance of the sensor by simulation using eigenmode expansion. We calculate that refractive index changes in the order of $10^{-6}$ RIU should be easily detectable for a component of length $10 \mu \mathrm{m}$. Moreover, we illustrate that the operation wavelength of the sensor can be easily tuned to a desired wavelength for a wide range of environmental refractive indices, making our device suitable for chemo- and biosensing.
\end{abstract}

(C) 2006 Optical Society of America

OCIS codes: (130.3120) Integrated Optics Devices; (130.6010) Sensors; (240.6680) Surface Plasmons

\section{References and links}

1. Homola J, "Present and Future of Surface Plasmon Resonance Biosensors," Anal. Bioanan. Chem., 377, 528 $539(2003)$

2. Čtyrocký J., Homola J, Lambeck PV, Musa S, Hoekstra HJWM, Harris RD, Wilkinson JS, Usievich B, Lyndin NM, "Theory and Modelling of Optical Waveguide Sensors Utilising Surface Plasmon Resonance," Sens. Actuators B,54, 66 - 73 (1999)

3. Harris Rd, Wilkinson JS, "Waveguide Surface Plasmon Resonance Sensors," Sens. Actuators B,29, 261 - 267 (1995)

4. Homola J, Čtyrocký J, Skalský M, Hradilová J and Kolářová P, “A Surface Plasmon Resonance Based Integrated Optical Sensor," Sens. Actuators B,38-39, 286 - 290 (1997)

5. Dostálek J, Čtyrocký J, Homola J, Brynda E, Skalský M, Nekvindová P, Špiriková J, Škvor J and Schröfel J, "Surface Plasmon Resonance Biosensor based on Integrated Optical Waveguide," Sens. Actuators B, 76, 8 - 12 (2001)

6. Bogaerts W, Baets R, Dumon P, Wiaux V, Beckx S, Taillaert D, Luyssaert B, Van Campenhout J, Bienstman P, Van Thourhout D, "Nanophotonic Waveguides in Silicon-on-Insulator Fabricated with CMOS Technology," J. Lightwave Technol., 23(1), 401-412 (2005)

7. Luff B, Wilkinson J, Piehler J, Hollenbach U, Ingenhoff J and Fabricius N, “ Integrated Optical Mach-Zehnder Biosensor," J. Lightwave Technol., 16, 583-592 (1998)

8. Prieto F, Sepúlveda, Calle A, Llobera A, Domínguez C, Abad A, Montoya A and Lechuga L M, "An Integrated Optical Interferometric Nanodevice based on Silicon Technology for Biosensor Applications," Nanotechnology, 14, 907-912 (2003)

9. Handbook of Optical Constants of Solids, edited by E. Palik, Academic Press New York (1985)

10. Nikolajsen T, Leosson K, Salakhutdinov I and Bozhevolnyi S, "Polymer-based Surface-Plasmon-Polariton Stripe Waveguides at Telecommunication Wavelengths," Applied Physics Letters, 82, 668-670 (2003)

11. Hochberg M, Baehr-Jones T, Walker C and Scherer A, "Integrated Plasmon and Dielectric Waveguides," Opt. Express, 12 5481-5486 (2002)

$\# 71072$ - \$15.00 USD

(C) 2006 OSA
Received 18 May 2006; revised 17 July 2006; accepted 19 July 2006

7 August 2006 / Vol. 14, No. 16/ OPTICS EXPRESS 7063 
12. Homola J, Sinclair S and Gauglitz G, "Surface Plasmon Resonance Sensors: Review," Sens. Actuators B, 54, 3-15 (1999)

13. Bueno FJ, Esteban O, Díaz-Herrara N, Navarrete MC and González-Cano A, "Sensing Properties of Asymmetric Double-Layer-Covered Tapered Fibers," Applied Optics, 43, 1615-1620 (2004)

14. CAMFR, http://sourceforge.org/projects/CAMFR

15. Bienstman P and Baets R,"Optical Modelling of Photonic Crystals and VCSELs using Eigenmode Expansion and Perfectly Matched Layers," Opt. Quantum Electron 33, 327-341 (2001)

16. Debackere P, Bienstman P, Baets R ,"Improved ASR Convergence for the Simulation of Surface Plasmon Waveguide Modes," OWTNM 2006 Proceedings, 14 (2006)

17. Debackere P, Bienstman P, Baets R, "Improved ASR Convergence for the Simulation of Surface Plasmon Waveguide Modes," to appear in special issue of Optical and Quantum Electronics on Optical Waveguide Theory and Numerical Modeling (2006)

\section{Introduction}

The use of surface plasmon resonance (SPR) for biological and chemical sensing is well established [1]. The high sensitivity of this technique to surface phenomena makes it ideal for use in real-time and label-free biosensors where very small changes in refractive index must be detected. Driven by the vision of a laboratory on a chip and its impact in numerous applications such as detection, biosensing, kinetic and binding studies and point-of-care diagnostics, extensive work has been done to miniaturize SPR biosensors. In the past decade, several integrated optical SPR sensors have been demonstrated [2, 3, 4], in which thin gold films serving as a platform for the attachment of sensing films are deposited on top of an integrated optical waveguide system. However, all integrated SPR sensors that have been investigated so far are fabricated in a material system with a low refractive index contrast $[2,3,4,5]$, keeping typical dimensions of waveguides and optical components too large for miniaturization and consequent lab on chip applications. Working with a high refractive index material system such as silicon-on-insulator is a more straight-forward approach to meet the requirements for high-level integration and high-throughput fabrication.

A high index contrast material system puts fundamental limits to the resonant excitation of surface plasmons at a gold-water surface. Phase-matching of guided waveguide modes with surface plasmon modes can be obtained, but only for modes with an effective index that is much lower than the refractive index of the waveguide. Moreover, the operating spectral range of such a device will be limited and set by the conditions for phase-matching, as is the case in conventional SPR waveguide sensors.

In this paper, we propose a novel configuration for a biosensor in silicon-on-insulator (SOI) [6]. The basic element of our sensor is a surface plasmon interferometer consisting of a thin layer of gold embedded in the silicon membrane. The working principle of this interferometer will be outlined in detail in Section 2. In Section 3, we show that our sensor has a highly customizable behavior. We define a set of design parameters that allow to tune the operation of our sensor to a desired wavelength range and/or to a desired range of analyte refractive indices. Finally, in Section 4, we investigate the sensitivity of our sensor for bulk refractive index changes and for the addition of thin dielectric films representative of protein layers.

\section{Theory}

Using interference as a a means to detect refractive index changes is not new, and integrated sensors using this principle have been realized in low-index [7] and high-index material systems [8]. Their working principle is based on the fact that refractive index variations induce a phaseshift in one of the arms of the Mach-Zehnder interferometer (MZI), this phase-shift results in an intensity variation in the output waveguide.

$$
I \propto[1+V \cos (\Delta \phi)]
$$

$\# 71072$ - \$15.00 USD

(C) 2006 OSA
Received 18 May 2006; revised 17 July 2006; accepted 19 July 2006 7 August 2006 / Vol. 14, No. 16 / OPTICS EXPRESS 7064 
where $\Delta \phi=\left(\phi_{r}-\phi_{s}\right)$ is the phase shift between guided modes in the sensing arm and the reference arm. The visibility factor $(V)$ gives the contrast between the maximum and the minimum transmitted intensity and depends on the coupling factor of the divisor and the propagation losses of guided modes in the interferometer arms.

The same principles can be applied to a surface plasmon interferometer. A sketch of our device is depicted in Fig. 1. The interferometer consists of a gold layer (refractive index taken from [9]) embedded into the silicon membrane $(n=3.45)$ on top of a supporting silica layer $(n=1.45)$, all dimensions and length scales are depicted in the figure. The high degree of asymmetry associated with the gold layer (top interface $n \propto 1.33$, bottom interface $n=3.45$ ) assures that the surface plasmon modes associated with the upper and lower of the metal-dielectric interfaces will never be able to couple, their wavevectors differ too much. So the gold layer possesses two distinct surface-plasmon modes which propagate through the structure without influencing each-other. Exciting these modes is done by end-fire coupling from a regular SOI waveguide with the transverse-magnetic ground mode $[10,11]$. So one can see that this device is in fact an interferometer. Two independently propagating surface plasmon modes are excited at the beginning of the gold layer, they propagate independently through the structure, the phase of the top surface plasmon mode is influenced by the refractive index of the environment, while the phase of the bottom surface plasmon mode is insensitive to any refractive index changes. At the end of the gold layer both surface plasmon mode excite the ground mode of the SOI waveguide, depending on the relative phase of the surface plasmon modes their contributions to the ground mode will interfere constructively or destructively.

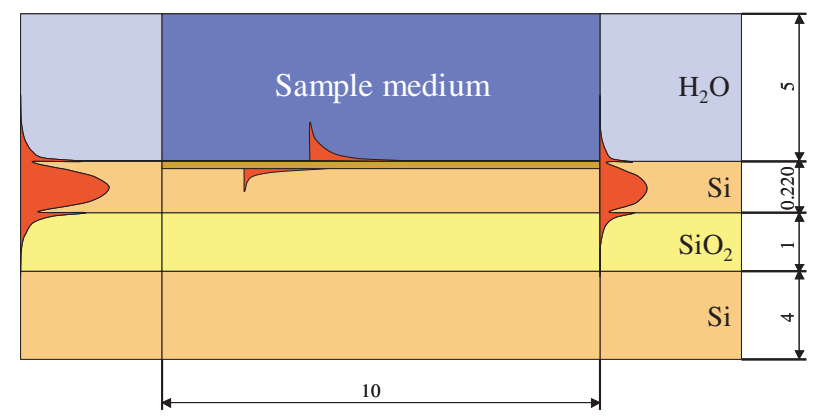

Fig. 1. Schematical setup of the proposed structure, all dimensions in $\mu \mathrm{m}$

To have a good interferometer both 'arms' should be single mode waveguides. Only two different modes should be allowed to propagate through the structure, the sensing surface plasmon mode at the top and the reference surface plasmon mode at the bottom. For the upper path it is evident that the surface plasmon mode at the gold-dielectric (in this case: water-) interface is the only guided mode that is supported. For the lower path this is less evident. However, one should take into account that the presence of the gold layer influences the cut-off properties of the silicon slab waveguide. Figure 2 plots the effective indices of all guided TM modes of a silicon membrane slab waveguide with and without a thin $60 \mathrm{~nm}$ gold layer on top. While the unclad waveguide has an index-guided mode for all thicknesses considered in this plot, the gold-clad waveguide has a cutoff thickness for index-guided modes of about $230 \mathrm{~nm}$. For all thicknesses below this value, only the internal surface plasmon mode remains. So if we chose the thickness of the silicon layer lower than $230 \mathrm{~nm}$, the only two modes that exist in the goldclad waveguide section are the internal and the external surface plasmon wave, ensuring that both 'arms' of the interferometer are monomode.

A second important contribution to the visibility $V$ is the coupling between the incoming

\#71072 - \$15.00 USD

(C) 2006 OSA
Received 18 May 2006; revised 17 July 2006; accepted 19 July 2006

7 August 2006 / Vol. 14, No. 16 / OPTICS EXPRESS 7065 
waveguide mode and the modes that propagate in both 'arms'. In our case this means that we have to calculate the coupling efficiency of the fundamental TM mode in the silicon waveguide to the two surface plasmon modes at the upper and lower gold interfaces. Coupling losses between the two regions were calculated by evaluating the overlap integrals of the modes in question. The coupling losses are plotted in Fig. 3 for different configurations of the sensing section. When the gold layer is not embedded but simply put on top of the silicon membrane, the coupling efficiency to the upper and lower surface plasmon modes differ strongly. However, by embedding the gold layer into the silicon membrane, the coupling efficiency can be adjusted over a very large range. Although it is possible to divide the input light equally over both branches of the interferometer, due to the difference in propagation loss of both surface plasmon modes this is not desirable.

Dispersion guided modes as a function of Si thickness

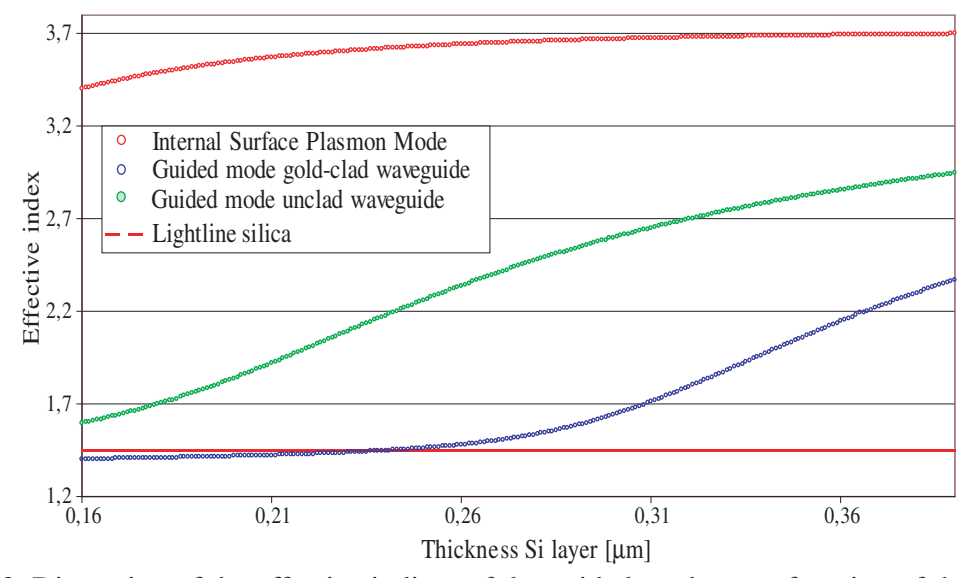

Fig. 2. Dispersion of the effective indices of the guided modes as a function of the waveguide thickness. The presence of the gold layer on top of the waveguide shifts the effective index of the index-guided mode to lower values. For a waveguide thickness of $230 \mathrm{~nm}$ the effective index of this mode is smaller than the refractive index of $\mathrm{SiO}_{2}$ so that this mode starts to radiate into the silica. For thicknesses below $230 \mathrm{~nm}$ the gold-clad $\mathrm{Si}$ waveguide has no index-guided modes.

Figure 4 illustrates the interferometric nature of our device. For a sensing section of length $10 \mu \mathrm{m}$, the transmitted intensity of the fundamental TM mode of the silicon slab waveguide is plotted as a function of refractive index of the sample medium. For this simulation, we have chosen a wavelength of $1.55 \mu \mathrm{m}$, which is in the near-infrared region and suitable for biosensing applications. When the upper and lower surface plasmon modes arrive in phase at the end of the sensing section, constructive interference leads to maximal transmission. However, for certain values of the sample refractive index, the phase difference between the two modes equals $\pi$, resulting into destructive interference and a minimum in the transmission curve.

Although the theory as outlined above has been presented here for a fixed wavelength and variable refractive index of the sample medium, there is a second, and perhaps more useful, method of using this device. As outlined above, the first method is to use a monochromatic input mode and monitor the output power as a function of the refractive index of the sample. This detection approach is commonly called 'intensity measurement mode' [12]. The second mode of operation uses a broadband input mode and as a function of the refractive index of the sample medium we monitor the position of the spectral minima in the transmission curve. This approach has been called 'wavelength interrogation mode' in the literature [12]

$\# 71072$ - \$15.00 USD

(C) 2006 OSA
Received 18 May 2006; revised 17 July 2006; accepted 19 July 2006 7 August 2006 / Vol. 14, No. 16 / OPTICS EXPRESS 7066 


\section{Coupling Loss as a function of Si thickness}

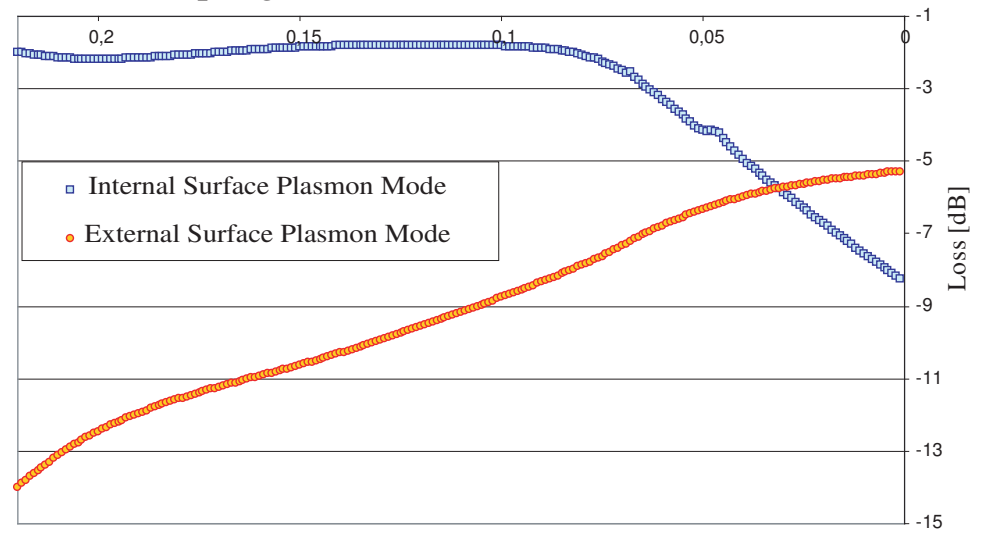

Thickness Si layer $[\mu \mathrm{m}]$

Fig. 3. Coupling loss to surface plasmon modes as a function of the waveguide thickness. The input waveguide has a thickness of $220 \mathrm{~nm}$, the thickness of the Si layer supporting the gold layer varies from $220 \mathrm{~nm}$ (Si layer is equally thick as the Si layer in the input waveguide) to $0 \mathrm{~nm}$ (Au layer is on top of the $\mathrm{SiO}_{2}$ layer).

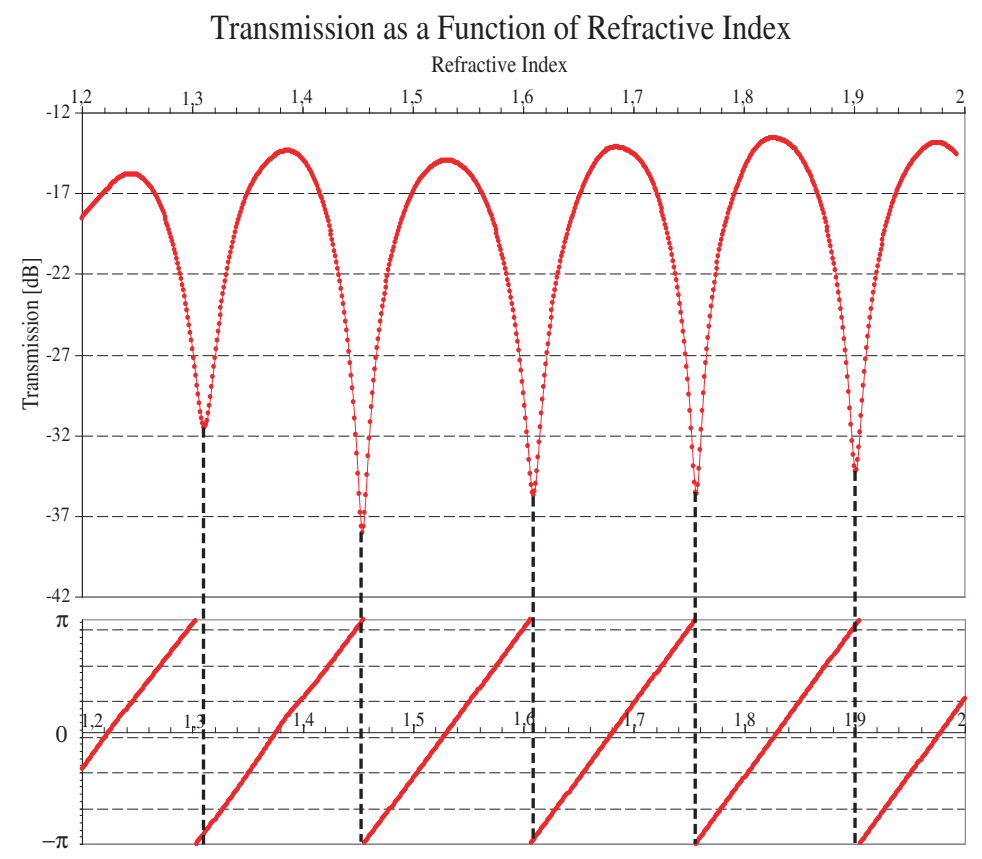

Fig. 4. Transmission of the structure depicted in Fig. 1. The length of the structure is 10 $\mu \mathrm{m}$

In Fig. 5 we have simulated the response of the structure shown in Fig. 1 to a broadband incoming waveguide mode. The refractive index of the sample medium is fixed at a value of 1.33. This behavior can also be explained by comparing the phase difference between the

$\# 71072$ - \$15.00 USD

(C) 2006 OSA
Received 18 May 2006; revised 17 July 2006; accepted 19 July 2006

7 August 2006 / Vol. 14, No. 16 / OPTICS EXPRESS 7067 
internal and the external surface plasmon modes as can be seen in the bottom of Fig. 5.

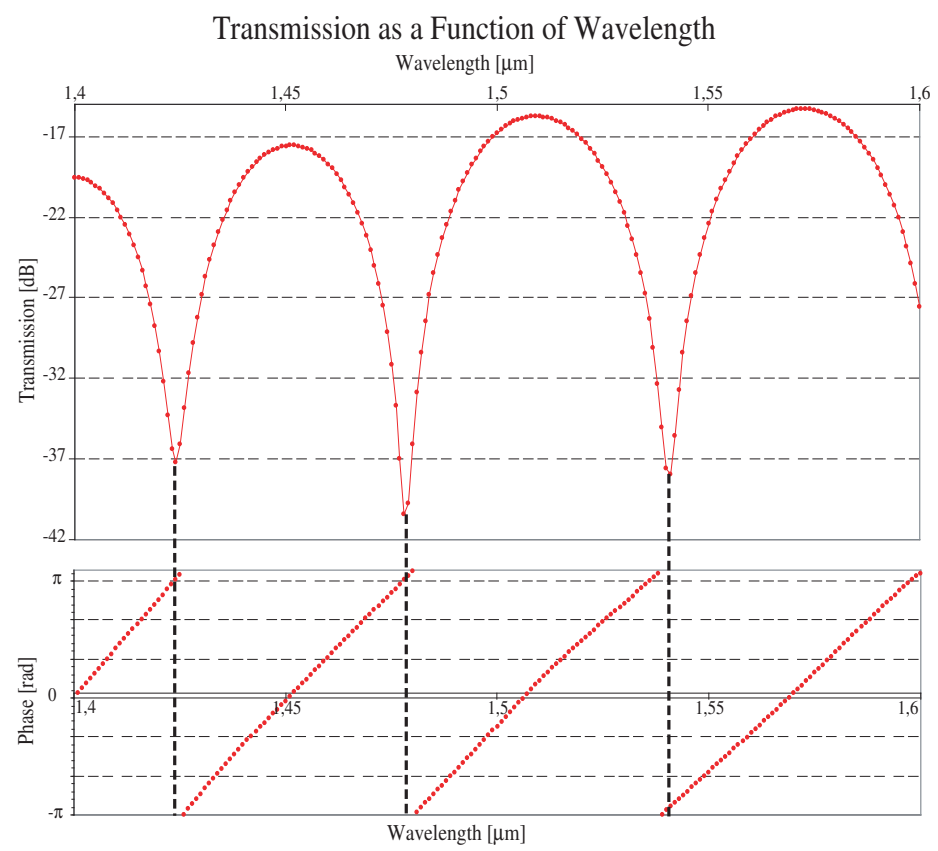

Fig. 5. Transmission of the structure as a function of the wavelength

In both modes of operation a sensor length of approximately $10 \mu \mathrm{m}$ should suffice, which is two orders of magnitudes smaller than current integrated surface plasmon sensors.

\section{Design Principles}

The resonance wavelength or resonance refractive index of SPR sensors can be tuned to some extent by using buffer layers between the dielectric waveguide layer and the gold layer or by choosing the appropriate metal[2, 4, 5, 13]. The fact that our device is based on interference rather than phase-matching allows one to tune the response of the device much more easily. There is no need for additional buffer layers, or a different metal to achieve the appropriate behavior, one simply has to modify the geometry of the device.

As will be shown below the device is characterized by three important design parameters. In the following section we will describe the design parameters, to establish some design rules for this device.

The first, and perhaps most important design parameter is the length of the sensing region. By varying the length one can tune the visibility $V$, and the position of the peaks. However, as will be shown below, the length of the device has to satisfy two different equations.

The wavelength ,or refractive index of the sample-medium, for which the transmission is minimal are completely determined by the following equation

$$
\begin{aligned}
& \mid\left(\phi_{T(\text { intern,in })}+\phi_{\text {prop, intern }}+\phi_{T(\text { in, intern })}\right)- \\
& \left(\phi_{T(\text { extern,in })}+\phi_{\text {prop, }, \text { extern }}+\phi_{T(\text { in, }, \text { xtern })}\right) \mid \approx \pi,
\end{aligned}
$$

where $\phi_{T(\text { intern,in })}$ is the phase difference due to the coupling of the incoming silicon waveguide mode to the internal plasmon mode, $\phi_{\text {prop, intern }}$ is the phase due to the propagation of the internal plasmon mode along the sensing region, and $\phi_{T(\text { in,intern })}$ is the phase difference due to

$\# 71072$ - \$15.00 USD

(C) 2006 OSA
Received 18 May 2006; revised 17 July 2006; accepted 19 July 2006

7 August 2006 / Vol. 14, No. 16 / OPTICS EXPRESS 7068 
the coupling of this internal plasmon mode to the silicon waveguide mode. Because of reciprocity $\phi_{T(\text { intern, in })}=\phi_{T(\text { in,intern })}$. Similar definitions hold for the external plasmonmode. Using this equation one can easily tune the position of minimal transmission to a desired frequency or refractive index range.

To achieve total destructive interference the losses along each path should be the same, so that at the end both interacting modes carry the same amount of power. These conditions ensure that the visibility $V$ will be maximal. Depending on the wavelength or refractive index for which one would want the transmission to reach a minimum, it is quite straightforward to prove that the optimal length is given by

$$
L=\frac{1}{k_{-}^{\text {external }}-k_{i}^{\text {internal }}} \frac{1}{\log e} \log \left(\frac{\mid\left. T(\text { intern }, \text { in })\right|^{2}}{\mid\left. T(\text { extern }, \text { in })\right|^{2}}\right),
$$

where $k_{i}^{\text {internal }}$ and $k_{i}^{\text {external }}$ are the imaginary parts of the $k$-vector of the internal and the external plasmonmode, $T$ (intern, in) and $T$ (extern, in) are the transmission coefficients of the incoming dielectric mode to the internal and the external plasmon modes at the chosen frequency or refractive index for which the transmission should be minimal.

Equation 2 provides us with a multitude of possible lengths corresponding to the order of the interference effect, equation 3 only provides us with one single possible length. By varying only the length of the device it is impossible to satisfy both equations at the same time. This automatically brings us to the other two design parameters, namely the coupling sections and the thickness of the gold layer.

For a given wavelength or refractive index, one can see from equation 3 that it is possible to change the length for which the power along both paths is the same. In order to do so we have to change the values of $\mid\left. T($ extern, in $)\right|^{2}$, and $\mid\left. T($ intern, in $)\right|^{2}$, which can be done by reducing the thickness of the silicon layer in the sensing region (as can be seen on Fig. 3). This provides us with an extra design parameter, which allows us to satisfy both equations more accurately.

Enhancing the coupling to the external plasmon mode, and in doing so minimizing the ratio $\frac{\mid\left. T(\text { extern, in })\right|^{2}}{\mid\left. T(\text { intern, in })\right|^{2}}$, results in other significant benefits, such as a smaller sensor length, an increased sensitivity of the sensor, and an overall higher transmission.

There is one fundamental limit however. Since the inner plasmon mode is propagating in a high index region its intrinsic loss will always be higher than the loss of the external plasmon mode (for a wavelength of $1.55 \mu \mathrm{m}$, and a refractive index of 1.2 , the loss of the external plasmon mode is equal to $0.04 \mathrm{~dB} / \mu \mathrm{m}$ while the loss of the internal plasmon mode equals $1.79 \mathrm{~dB} / \mu \mathrm{m})$. Because of this the coupling to the inner mode should always be greater than the coupling to the outer mode. However, simulations show that this is not a major drawback as the sensitivity of the structure is up to par with current sensors, even though only half the incoming power is affected by refractive index changes.

If the above mentioned equations are not yet fully satisfied we can also change the thickness of the gold layer. The effects of changing the thickness of the gold layer are relatively small compared to the effect of other design parameters, but they are large enough to allow us to satisfy both equations.

Using these simple rules we have optimized the sensor depicted in Fig. 1 so that the transmission would be minimal for a refractive index of the sample medium of 1.33 , and a wavelength of $1.55 \mu \mathrm{m}$. In the optimized design the thickness of the silicon membrane is equal to $101 \mathrm{~nm}$, and the length of the device is equal to $6.055 \mu \mathrm{m}$. Simulation results for this device are shown in Fig. 6. 


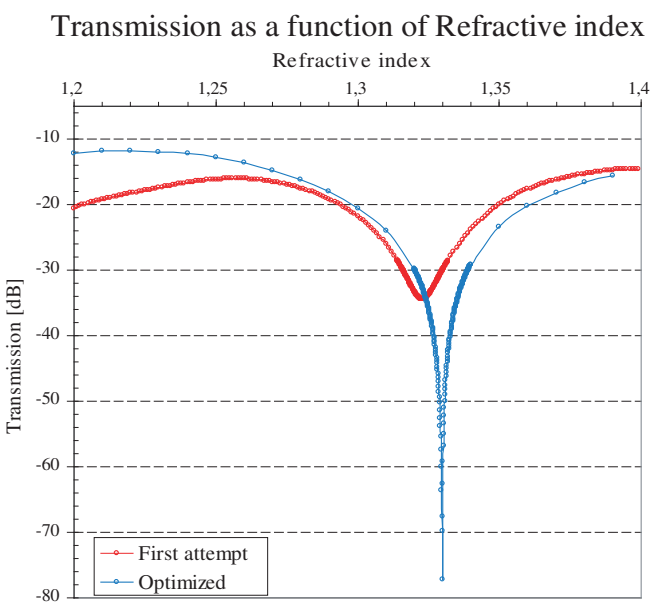

Fig. 6. Simulation results for the optimized structure

\section{Sensitivity}

\subsection{Simulation}

We investigated the sensitivity of our sensor to bulk refractive index changes and its sensitivity to the addition of a thin dielectric film representative of a protein layer. For all our simulations, we used CAMFR [14], an in house-developed eigenmode solver [15]. The calculation algorithm consists of two steps. A Fourier Modal Method algorithm, which was recently improved by incorporating adaptive spatial resolution in the method[16, 17], generates estimates. These estimates are then refined using the mode-matching technique. The reference data for the refractive index of gold was taken from [9]

\subsection{Sensitivity}

If we take the intensity measurement approach to detect refractive index changes we can calculate that the sensitivity for this device reaches values of $10000 \mathrm{~dB} / R I U$ (refractive index unit). In conjunction with an optoelectronic system which can measure changes in the optical power of $0.01 \mathrm{~dB}$, variations in the refractive index as small as $10^{-6}$ can be measured. Prism coupled SPR sensors with detection limits of $1 \times 10^{-5}$ have been demonstrated, value taken from [12]), while grating coupled sensors typically have a detection limit of $5 \times 10^{-5}$ RIU. Integrated sensors based on the MZI configuration display measured detection limits of $7 \times 10^{-6}$ RIU [8], while integrated surface plasmon resonance sensors in low-index contrast material systems achieve measured values of $2000 \mathrm{~dB} / \mathrm{RIU}$, this corresponds to a detection limit of $5 \times 10^{-6}$ RIU [4]. However, the dimensions of our device are two orders of magnitude smaller than the aforementioned devices. This means that the smallest amount of a certain molecule that can be detected will also be two orders of magnitude smaller than current integrated surface plasmon sensors.

Taking the wavelength interrogation approach, sensitivity is defined as the shift of the wavelength for which transmission is minimal as a function of the refractive index of the sample medium $(\Delta \lambda / R I U)$. The value of the sensitivity can be derived from Fig. 7 .

From Fig. 7 one can see that the shift of the wavelength for which transmission is minimal as a function of the refractive index of the sample medium is equal to $463.5 \mathrm{~nm}$ per refractive

$\# 71072$ - \$15.00 USD

(C) 2006 OSA
Received 18 May 2006; revised 17 July 2006; accepted 19 July 2006

7 August 2006 / Vol. 14, No. 16 / OPTICS EXPRESS 7070 
Transmission minimum as a function of bulk refractive index

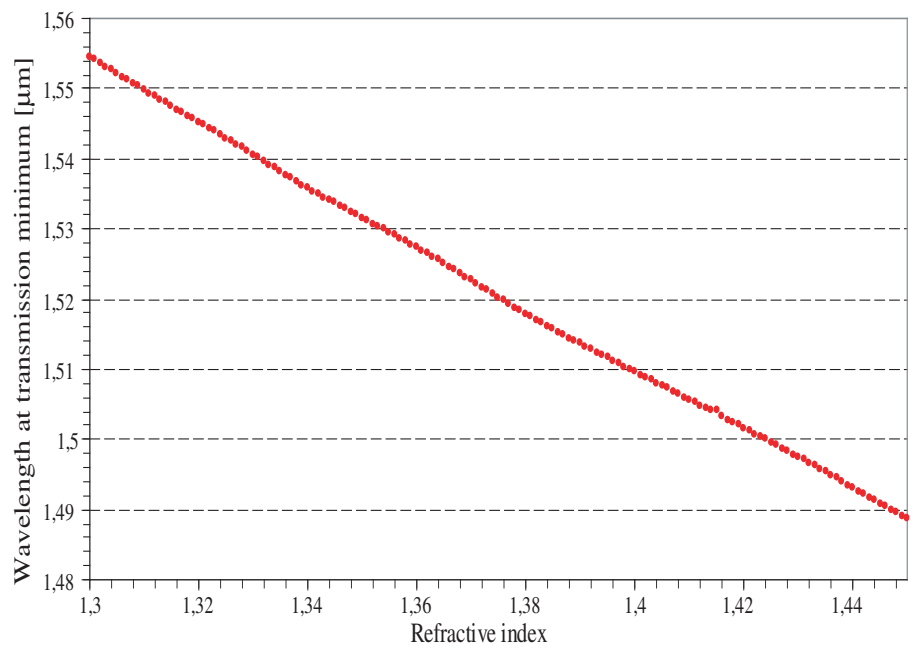

Fig. 7. Shift of the wavelength for which transmission is minimal as a function of the refractive index of the sample medium

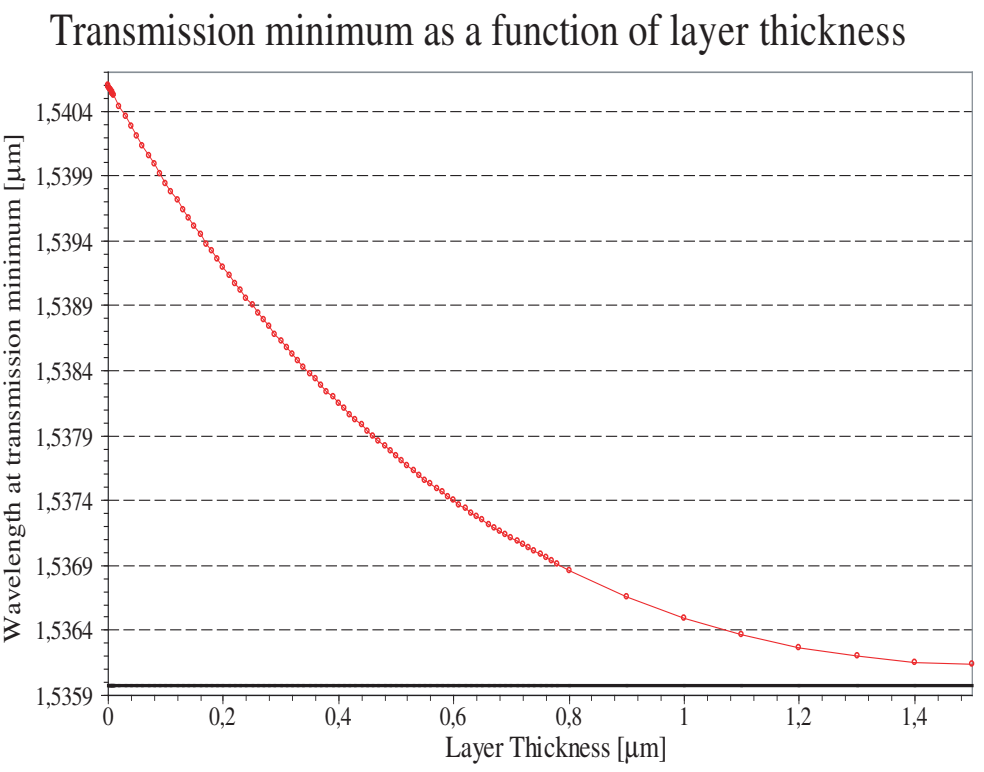

Fig. 8. Shift of the wavelength for which transmission is minimal as a function of the thickness of the absorbed layer

index unit. According to [12] a prism based sensors has a shift of $13800 \mathrm{~nm}$ per refractive index unit at $850 \mathrm{~nm}$, an a grating based device has a value of $630 \mathrm{~nm}$ per refractive index unit.

In order to demonstrate that our proposed device can detect very thin dielectric layers representative of thin protein layers, we have determined the shift of the wavelength for which the transmission is minimal as a function of the thickness of an adsorbed layer at the $A u$-sample 
medium interface. In Fig. 8 the adsorbed layer thicknesses varyies from 1 to $400 \mathrm{~nm}$ and has a refractive index of 1.34. By inspection of the slope of the curve we can estimate the dependence of the peak position on the layer thickness to be approximately equal to $6 \mathrm{pm} / \mathrm{nm}$. This demonstrates that our device can be used to measure layer thicknesses of abso rbed protein layers.

\section{Conclusions}

We have presented in this paper a novel concept for a biological sensor using surface plasmon waves. The new device was described from a theoretical point of view, and simulation results show its potential for sensing applications. We have layed out a set of theoretical design parameters and have shown that the performance of this device can be greatly improved following these design parameters.

Due to its different working principle as compared to other devices, this device has a number of interesting benefits. Firstly, the device is two orders of magnitude smaller than conventional surface plasmon waveguide sensors, due to the integration into a high-index contrast material system. Secondly the device is highly tunable, making an excellent candidate for a vast number of applications.Thirdly, the sensitivity of this device is comparable with that of state of the art biological sensors.

The authors believe this novel concept to be an important step toward a fully integrated surface plasmon lab-on-chip solution.

\section{Acknowledgments}

This work was carried out in the context of the GOA project (Ghent University), and was supported by the Belgian IAP PHOTON network. S. Scheerlinck thanks the Institute for the Promotion of Innovation through Science and Technology in Flanders (IWT-Vlaanderen) for a scholarship. P. Bienstman acknowledges the Flemish Fund for Scientific Research (FWOVlaanderen) for a postdoctoral fellowship.

\#71072 - \$15.00 USD

(C) 2006 OSA
Received 18 May 2006; revised 17 July 2006; accepted 19 July 2006 7 August 2006 / Vol. 14, No. 16 / OPTICS EXPRESS 7072 